\title{
Spectral Models of Kuiper Belt Objects and Centaurs
}

\author{
Dale Cruikshank \\ NASA Ames Research Center \\ Cristina M. Dalle Ore \\ NASA Ames Research Center and SETI Institute, Mountain View, CA
}

June25, 2003

\begin{abstract}
We present models of the spectral reflectances of groups of outer Solar System objects defined primarily by their colors in the spectral region $0.4-1.2 \mu \mathrm{m}$, and which have geometric albedo 0.04 at wavelength $0.55 \mu \mathrm{m}$. Our models of the groups with the strongest reflectance gradients (reddest colors) use combinations of organic tholins. We test the hypothesis that metal-reddened igneous rock-forming minerals contribute to the red colors of Centaurs and KBOs by using the spaceweathered lunar soil as one of the components of our models. We find that our models can admit the presence of moderate amounts of space-weathered (metalreddened) minerals, but that they do not require this material to achieve the red colors of the reddest outer Solar System bodies. Our models with organic tholins are consistent with the results of other investigators.
\end{abstract}

\section{INTRODUCTION}

The compositions of the small bodies in the outer Solar System (OSS), particularly those with geometric albedo $=0.1$ at $\lambda=0.5 \mu \mathrm{m}$, are understood only in broad outline. While many outer belt asteroids, small planetary satellites, Centaur objects, Kuiper Belt objects (KBOs), and comet nuclei have been observed photometrically and spectroscopically (primarily at wavelengths $0.3 \leq \lambda \leq 2.5 \mu \mathrm{m}$ ), relatively little direct information on their compositions has resulted. The natural materials expected to compose these bodies are ices of $\mathrm{H}_{2} \mathrm{O}$ and other volatile molecules, minerals, and solid macromolecular carbonaceous material. The term "macromolecular carbonaceous" includes, but is not limited to, the complex organic materials found in the carbonaceous meteorites (Cronin et al. 1988, Cronin and Chang 1993), interplanetary dust particles (Bradley et al. 1996), and micrometeorites (Maurette et al. 1996). It also includes complex organic materials presumed to have been synthesized by the deposition of energy in CHON-rich matter directly on the surfaces of KBOs and other solid bodies in the outer Solar System.

Absorption bands of $\mathrm{H}_{2} \mathrm{O}$ ice have been detected in a few Centaurs (e.g., Brown et al. 1998), Kuiper Belt objects (e.g., Brown et al. 1999), irregular planetary satellites (Owen et al. 1999), and at least one comet

(C) 2003 Kluwer Academic Publishers. Printed in the Netherlands. 


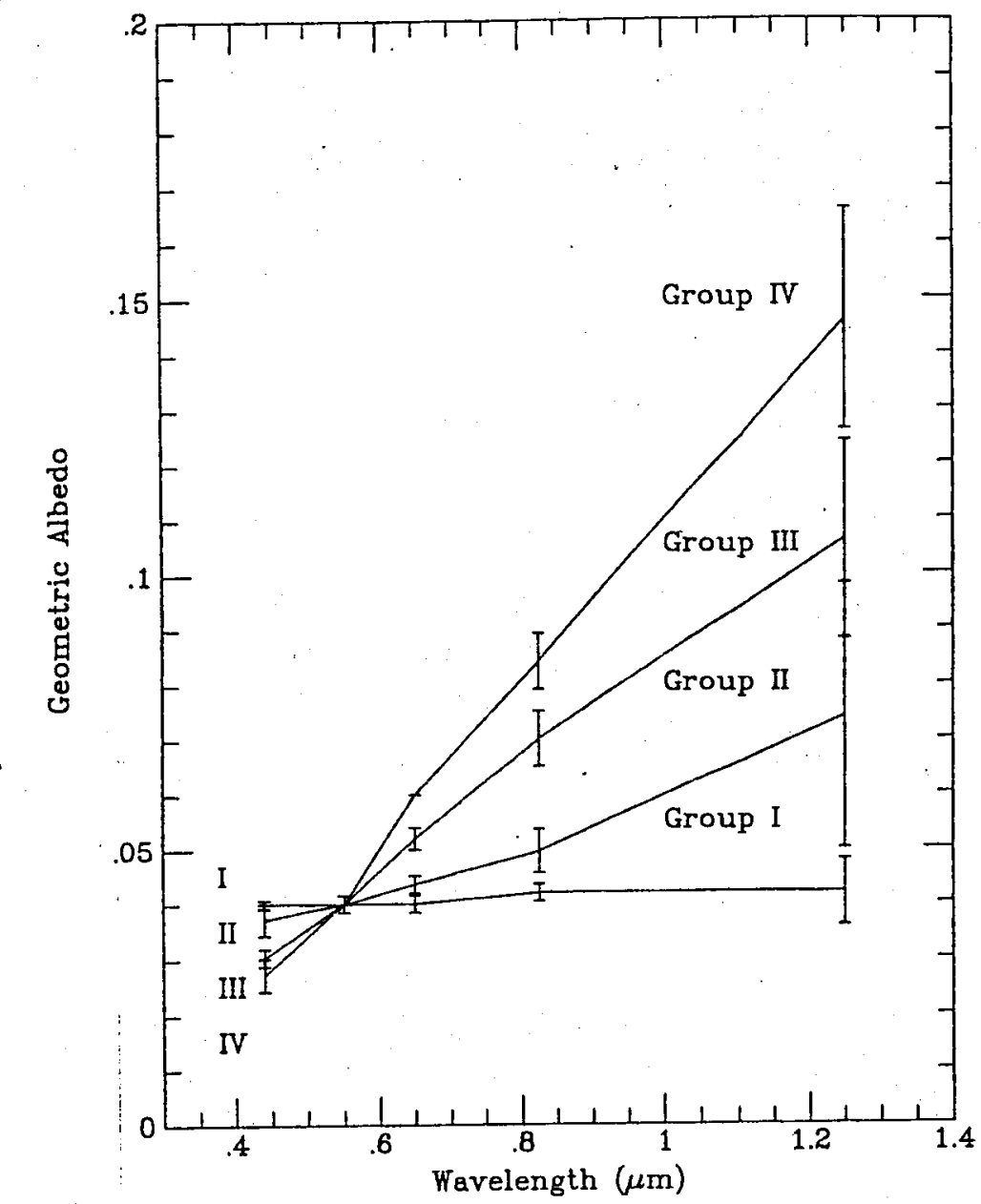

Figure 1. Four groups defined by photometry of Centaurs and Kuiper Belt objects by Barucci et al. (2001). Representative members of the groups are: Group I (1996 TO66, 2060 Chiron), Group II (1997 CU26, 1998 SG35), Group III (1993 SC, 1997 CQ29), Group IV (5145 Pholus, 1994 TB). We have normalized these curves to geometric albedo 0.04 at wavelength $0.56 \mu \mathrm{m}$.

\section{MODEL COMPONENTS}

\subsection{ORGANIC SOLIDS}

We are especially concerned in this paper with Solar System bodies having large reflectivity gradients, or red colors. When the very red Centaur object 5145 Pholus was first observed in the 0.3-1.0 $\mu \mathrm{m}$ region, three different investigator teams (Fink et al. 1992; Binzel 1992; Davies et al. 1993) all concluded that only solid organic materials, or materials similar to a solid polymer of hydrogen cyanide, could account for the red 
color. Simple spectrum matching with the reflectance of the synthetic organic material called tholin provided a reasonably good fit to the color of Pholus in that limited wavelength region, while plausible minerals and ices did not.

The Trojan asteroid 624 Hektor (classified as D) is among the reddest of the traditional asteroids, although it is much less red than the reddest of the KBOs and Centaurs. Cruikshank et al. (2001) showed that the spectral reflectance of Hektor can be modeled with Mg-rich pyroxene and serpentine, over the wavelength range $0.3-4 \mu \mathrm{m}$. Their result demonstrated that organic solids are not essential for modeling low-albedo surfaces of the degree of redness exhibited by Hektor. By analogy, KBOs and Centaurs that are comparable in albedo and color to Hektor can be modeled with minerals (see below).

In the models of the reddest KBOs and Centaurs described below, the principal component imparting the red color (increasing absorption toward the violet end of the reflectance spectrum) is tholin. Tholin is the name given to a class of complex organic solids produced by the irradiation of mixtures of cosmically abundant reducing gases and ice, and is independent of any assumptions of the detailed chemistry of the material. The literature of tholin production, chemistry, optical properties, and applicability to natural settings in the Solar System is slowly expanding with the growing recognition of the widespread occurrence of complex organic solid material in planetary environments (e.g., Cruikshank and Khare 2000).

Several kinds of tholin have been made and analyzed. The starting mixtures, both in the form of gases and solids, have been the principal variables, while the energy source has typically been a plasma discharge producing ultraviolet light and charged particles. Table I shows a summary of four tholins produced at Cornell University, having demonstrated relevance to planetary environments. Other laboratories are now producing tholin materials (e.g., Ramirez et al. 2002; Imanaka et al. 2003).

The Cornell University tholins, which are macromolecular complexes of polymers, polycyclic aromatic hydrocarbons, nitriles and nitrogen heterocyclic compounds (when $\mathrm{N}$ is included in the starting mixture), should be regarded as an intermediate product of the irradiation of simple ices and gases. As Thompson et al. (1987) showed, when they irradiated a methane clathrate (frozen $\mathrm{CH}_{4}+\mathrm{H}_{2} \mathrm{O}$ ), the initial reddening of the material eventually progressed to an overall darkening as the carbon was more completely dehydrogenated. Andronico et al. (1987) found similar results with organics synthesized in the laboratory by ion beam bombardment; the materials continued to darken with increasing ion fluence. Continued energy deposition in tholins is expected to 
Table I. Tholins For Which Complex Refractive Indices Are Measured

\begin{tabular}{|c|c|c|c|}
\hline Name & Starting Mixture & Energy Source & References \\
\hline $\begin{array}{c}\text { Titan tholin } \\
\ldots\end{array}$ & 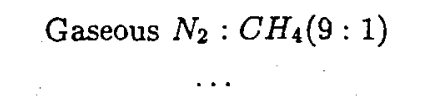 & Plasma Discharge & $\begin{array}{l}\text { Khare et al. 1984, } \\
\text { McDonald et al. } 1994\end{array}$ \\
\hline Triton tholin & Gaseous $N_{2}: C H_{4}(99.9: 0.1)$ & Plasma Discharge & McDonald etal. 1994 \\
\hline Ice tholin 1 & $\mathrm{H}_{2} \mathrm{O}: \mathrm{C}_{2} \mathrm{H}_{6}(6: 1)$ & Plasma Discharge & $\begin{array}{c}\text { Khare et al. } 1993, \\
\text { McDonald et al. } 1996\end{array}$ \\
\hline Ice tholin 2 & $\mathrm{H}_{2} \mathrm{O}: \mathrm{CH}_{3} \mathrm{OH}: \mathrm{CO}_{2}: \mathrm{C}_{2} \mathrm{H}_{6}$ & Plasma Discharge & McDonald et al. 1996 \\
\hline$\cdots$ & $(80: 16: 3.2: 0.8)$ & $\cdots$ & ‥ \\
\hline
\end{tabular}

remove hydrogen and other atoms, eventually resulting in a residue of mostly elemental carbon.

The tholins listed in Table I are important to the present work because their complex refractive indices ( $\mathrm{n}$ and $\mathrm{k}$ ) have been measured over a wavelength range comparable to that in which Solar System bodies have been observed. Rigorous scattering models require $\mathrm{n}$ and $\mathrm{k}$ of the modeled constituents for the radiative transfer calculations that yield synthetic spectra for comparison with observational data. Very few materials have been measured for $\mathrm{n}$ and $\mathrm{k}$ in the spectral region (and with the spectral resolution) that matches the observational data acquired from spacecraft and ground-based telescopes. Insofar as a few organic solids, the tholins, have been measured and chemically characterized, these materials have proven to be a suitable and plausible component of the successful models calculated to date. This success may be fortuitous, but probably it is not. The mechanisms for energy deposition in simple ices and gases are prevalent in planetary environments, and the existence of the requisite raw materials (CHON) is well established.

\subsection{Minerals}

In addition to ices and organic materials, some significant mineral component is expected in KBOs, particularly because olivine and pyroxene are seen in the dust of active comets (e.g., Wooden 2000). Cruikshank et al. (1998) found that Mg-rich olivine (Fo 82) was an important component in their models of 5145 Pholus, where its absorption near 
$1 \mu \mathrm{m}$ had a significant effect in shaping the steep red continuum of this Centaur.

Mafic minerals in the inner Solar System are "weathered" by the incident solar wind (e.g., Pieters et al. 2000), which has the effect of making their spectra substantially redder than the unaffected minerals exhibit. This alteration has been identified as solar wind sputtering, which selectively removes, reduces, and deposits the iron from mafic mineral grains as nanophase neutral iron on the undersides of adjacent grains (Hapke 2001). This process is now generally viewed as the cause of the red colors of Mercury, the Moon, and probably many of the mainbelt asteroids. Processes of space weathering in the OSS are expected to arise from other phenomena because of the intrinsic weakness of the solar wind beyond several Astronomical Units (AU). For example, galactic cosmic rays deposit approximately $160 \mathrm{eV}$ per molecule in the upper $100 \mathrm{~g} \mathrm{~cm}^{-2}$ of a planetary regolith at Pluto's distance AU (Johnson 1989), and recent estimates of the flux of anomalous cosmic rays are approximately a factor of 10 higher (Cooper et al. 2003). The effects on mineral grains in the surface zone of interaction (which may be tens of $\mathrm{cm}$ deep) can include sputtering, as well as grain amorphization (Brucato etal. 2003). The details of these processes in the OSS, and their effect(s) on the reflectance spectra of the materials in the optical surface remain to be studied.

In our models we test the hypothesis that metal-reddened mafic minerals contribute to the red colors of Centaurs and KBOs by using the space-weathered lunar soil as one of the components. Shkurator et al. (1999, Fig. 5) derived the optical constants for the reddened lunar soil by inverting their scattering model, and Dr. Shkuratov kindly provided the values of $n$ and $k$ that we use here.

\subsection{ICES}

Although ices are expected to be a major constituent of $\mathrm{KBOs}$ and Centaurs, the only ice identified with certainty on KBOs and Centaurs is $\mathrm{H}_{2} \mathrm{O}$, seen in the spectrum through its broad absorption bands at 1.55 and $2.0 \mu \mathrm{m}$, and the short wavelength part of the $2.7 \mu \mathrm{m}$ band (e.g., Brown et al. 1998, 1999; Cruikshank et al. 1998; Poulet et al. 2002). The spectrum of 5145 Pholus shows a band complex at $2.17 \mu \mathrm{m}$ that has been attributed to $\mathrm{CH}_{3} \mathrm{OH}$ ice (Cruikshank et al. 1998), but could be a light hydrocarbon. Crystalline (hexagonal) $\mathrm{H}_{2} \mathrm{O}$ ice can be distinguished from amorphous ice, which is stable only at low temperature $(\mathrm{T} \leq 90 \mathrm{~K}$ ) by its spectral signature, in particular the presence of a temperature-sensitive absorption band at $1.65 \mu \mathrm{m}$ that is unique to hexagonal ice (Grundy and Schmitt, 1998). When the $\mathrm{H}_{2} \mathrm{O}$ ice bands 
are only weakly present, as in the cases of the KBOs on which ice has been identified, it is often impossible to discern the $1.65 \mu \mathrm{m}$ band or the detailed profile of the $2.0 \mu \mathrm{m}$ band, and it is therefore unclear if the ice is crystalline or amorphous.

\section{MODELS OF KUIPER BELT OBJECTS AND CENTAURS}

\subsection{MOdELS WITH ORganiCS}

We have used the Shkuratov radiative transfer code (Shkuratov et al. 1999) for scattering in particulate surfaces to calculate models of groups II, III, and IV defined by Barucci et al. (2001), as shown in Figure 2. For each group we show three models with different proportions of the four basic components Titan Tholin, Ice Tholin II, amorphous carbon, and lunar soil. While the Barucci color data extend only to the $J$ wavelength $(1.22 \mu \mathrm{m})$, we have calculated the models to $2.5 \mu \mathrm{m}$ because additional color and spectroscopic data exist for some of the brighter KBOs and Centaurs.

For models without the lunar soil component, we find that we can match the spectral gradient and the albedo level for all three groups by varying the proportions and grain sizes of the two tholins. The addition of lunar soil still allows adequate fits to the data points but introduces curvature to the calculated spectrum, notably a broad absorption at $1 \mu \mathrm{m}$ where pyroxene and feldspar in the lunar soil have overlapping absorption bands. The second pyroxene band around $2 \mu \mathrm{m}$ also appears in calculated spectra with larger amounts of lunar soil. In the reddest spectra, Group IV, the models allow a substantial fraction of these space-weathered, reddened minerals, but the absorption bands introduced into the calculated spectra are not seen in any KBO or Centaur spectra published to date. The model spectrum with only the tholins and carbon for Group IV provide a smoother fit to the data points, and on to $2.5 \mu \mathrm{m}$. The situation is similar for Group III; reddened minerals are allowed, but produce a spectrum with broad features that are not seen in other data for KBOs and Centaurs. In the Group II spectra the absorption band structure shows much less contrast, as the calculated spectra begin to flatten to match the less colored objects defining this group. Group II admits a larger fraction of lunar soil than the previous groups, although the downward turn of the spectral reflectance at the shortest wavelengths appears to be inconsistent with the data for KBOs and Centaurs.

We conclude from these models that the full range of redness of KBO and Centaur reflectance spectra, as well as their generally low albedos, 
can be matched with synthetic spectra calculated from combinations of tholins and amorphous carbon. We find that the model spectra can admit, but do not require the addition of space-weathered, igneous rock-forming minerals.

We noted above the photometric study of several KBOs by McBride et al. (2003) in which the critical V and J wavelengths were measured simultaneously. In Figure 3 we show our best-fit models for three representative objects from McBride's list. These are models in which tholins provide the color and carbon adjusts the albedo. In the case of KBO $1993 \mathrm{SC}$ (top panel) we add $\mathrm{H}$ and $\mathrm{K}$ photometry from Hainaut and Desanti (2002) to show that the model fits the data satisfactorily.

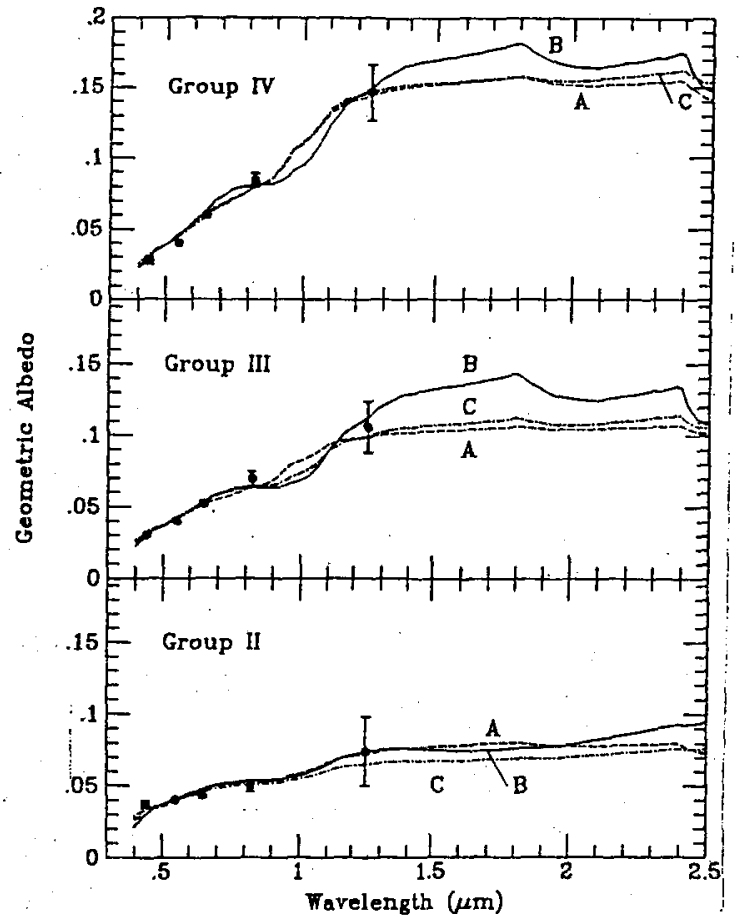

Figure 2. Shkuratov spatial (areal) mixture and intimate mixture models of groups II, III, and IV defined by Barucci et al. (2001). Key: IT2 = Ice Tholin 2, TT = Titan tholin, LS = space-weathered lunar soil, $A C=$ amorphous carbon. Grain sizes given in $\mu \mathrm{m}$ in parentheses. Top panel: Model A is $[0.36$ IT2 (10) +0.28 TT (10) $+0.36 \mathrm{AC}(10)]$. Model B is [0.48 IT2 (20) + 0.32 TT (30) + 0.2 LS (20)]. Model $\mathrm{C}$ is [0.35 IT2 (20) + 0.30 TT (10) + 0.15 LS (20) + 0.20 AC (10)]. Middle panel: Model A is [0.25 IT2 (10) + 0.20 TT (20) + 0.55 AC (10)]. Model B is 0.28 IT2 (20) $=0.42 \mathrm{TT}(60)+0.30 \mathrm{LS}(20)]$. Model $\mathrm{C}$ is $0.28 \mathrm{IT} 2(20)+0.24 \mathrm{TT}(30)+0.12$ LS (20) + 0.36 AC (30)]. Bottom panel: Model A is 0.20 IT2 (10) + 0.15 TT (30) + $0.65 \mathrm{AC}(10)]$. Model B is $[0.20 \mathrm{IT} 2(30)+0.06 \mathrm{TT}(80)+0.74$ LS (30)]. Model C is $[0.175 \mathrm{IT} 2(30)+0.065 \mathrm{TT}(30)+0.175 \mathrm{LS}(30)+0.585 \mathrm{AC}(30)]$. Each model is a spatial mix of TT and IT2, each intimately mixed with AC. ${ }^{1}$ 


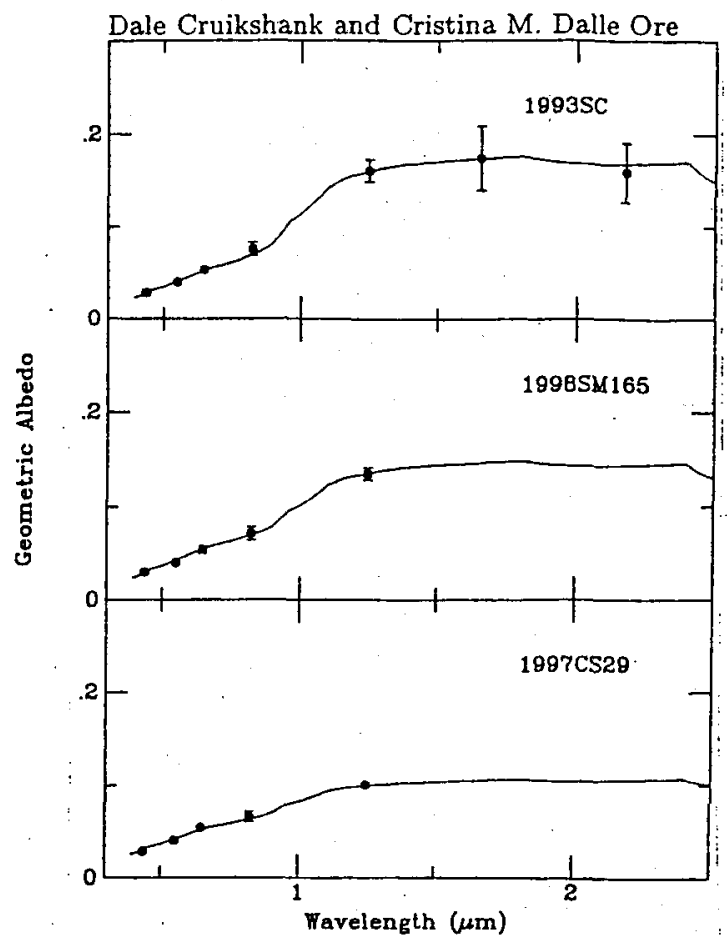

Figure 3. Shkuratov models of three objects from the McBride list. Each model is a spatial (area) mixture of two intimately mixed components; all of the particles are $10 \mathrm{~m}$ in size, and the numbers outside the brackets indicate the fraction of the area covered by a specific component. Key: $\mathrm{H}_{2} \mathrm{O}=$ water ice at temperature $40 \mathrm{~K}$, IT2 $=$ Ice Tholin 2, TT $=$ Titan tholin, $A C=$ amorphous carbon. $1993 \mathrm{SC}: 0.45$ [60\% $\mathrm{IT} 2+40 \% \mathrm{AC}]+0.55[70 \% \mathrm{TT}+30 \% \mathrm{AC}] .1998 \mathrm{SM} 165: 0.60[50 \% \mathrm{IT} 2+50 \%$ $\mathrm{AC}]+0.40[70 \% \mathrm{TT}+30 \% \mathrm{AC}] .1997 \mathrm{CS} 29: 0.50[50 \% \mathrm{IT} 2+50 \% \mathrm{AC}]+0.50$ $[40 \% \mathrm{TT}+60 \% \mathrm{AC}]$

Models of the kind described above can also incorporate $\mathrm{H}_{2} \mathrm{O}$ ice in amounts sufficient to display the $1.55,2.0$, and $2.5 \mu \mathrm{m}$ absorption bands. The discovery of $\mathrm{H}_{2} \mathrm{O}$ ice on planetary satellites, $\mathrm{KBOs}$, and Centaurs of geometric albedo (at $0.55 \mu \mathrm{m}$ ) 0.1 and less (e.g., Brown et al. 1998, 1999; Owen et al. 1999) demonstrates that the absorption bands are not entirely quenched by the coexistence of low-albedo materials on their surfaces. Indeed, in our models we find that we can include substantial amounts of $\mathrm{H}_{2} \mathrm{O}$ ice containing tholins as inclusions. The incorporation of tholins in the ice as inclusions is approximately equivalent to a mixture at the molecular level, and the optical constants of the mixture are calculated by effective medium theory (Shkuratov et al. 1999).

Figure 4 shows two sets of models of KBO 20000 Varuna, one without $\mathrm{H}_{2} \mathrm{O}$ ice, and one including $\mathrm{H}_{2} \mathrm{O}$ ice; the actual presence of ice on Varuna is uncertain. The observational data for Varuna in Fig. 4 are from McBride et al. (2003). 

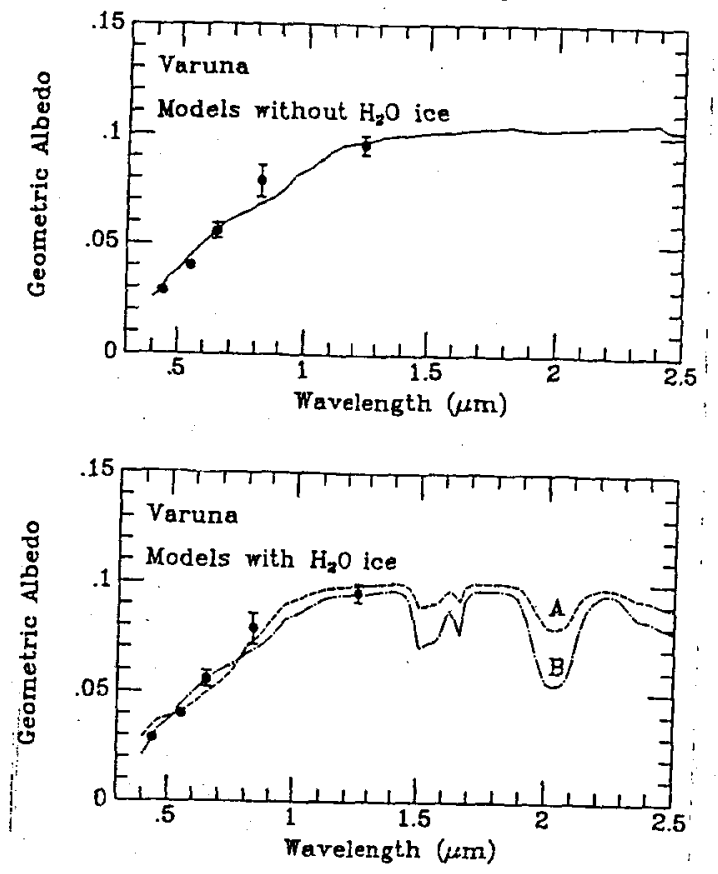

Figure 4. Representative models of Kuiper Belt object 20000 Varuna, showing a model without $\mathrm{H}_{2} \mathrm{O}$ ice (upper panel) and models including $\mathrm{H}_{2} \mathrm{O}$ ice in different abundances (lower panel). Each model is a spatial (area) mixture of two intimately mixed components. Key: $\mathrm{H}_{2} \mathrm{O}=$ water ice at temperature $40 \mathrm{~K}, \mathrm{IT} 2=$ Ice Tholin 2, $\mathrm{TT}=$ Titan tholin, $\mathrm{AC}=$ amorphous carbon. Grain sizes are given in $\mu \mathrm{m}$ in parentheses, and the numbers outside the brackets indicate the fraction of the area covered by a specific component. Upper panel: spatial mix of $60 \%$ of component 1 , which is an intimate mix of $[50 \%$ IT2 (10) $+50 \%$ AC (10)], and $40 \%$ of component 2 , which is an intimate mix of $[60 \% \mathrm{TT}(10)+0.4 \mathrm{AC}(10)]$. Lower panel: Model $A$ is $0.5\left[30 \% \mathrm{H}_{2} \mathrm{O}(10)\right.$ with inclusions amounting to $50 \% \mathrm{IT} 2$, intimately mixed with $70 \% \mathrm{AC}(15)]+0.5\left[80 \% \mathrm{H}_{2} \mathrm{O}\right.$ (10) with inclusions amounting to $50 \% \mathrm{TT}$, intimately mixed with $20 \% \mathrm{AC}(15)]$. Model $\mathrm{B}$ is $0.65\left[50 \% \mathrm{H}_{2} \mathrm{O}\right.$ (30) with inclusions amounting to $50 \%$ IT2, intimately mixed with $50 \% \mathrm{AC}(30)]+0.35\left[50 \% \mathrm{H}_{2} \mathrm{O}\right.$ (30) with inclusions amounting to $50 \% \mathrm{TT}$, intimately mixed with $50 \% \mathrm{AC}(30)$ ]

\subsection{MOdELS WiThOUT ORGANICS}

Objects having a moderate degree of redness, such as the D-type asteroids (mostly Trojans asteroids) and the objects in Groups I and II of Barucci et al. (2001) do not require the presence of organic materials to achieve their red colors. Cruikshank et al. (2002) modeled the spectrum of Trojan asteroid 624 Hektor with simple Hapke scattering models using only mafic minerals (Mg-rich pyroxene) and amorphous carbon. Their models included the spectral region out to $\lambda=3.6 \mu \mathrm{m}$, which covers the region of absorption bands in hydrous silicates; The models demonstrated that a significant amount of hydrous silicates 
(e.g., serpentine) can be present on Hektor without causing a $3 \mu \mathrm{m}$ absorption band that is detectable with data of modest quality. Figure 5, adapted from Cruikshank et al. (2001) shows the data for Hektor and a suite of spectral reflectance models using intimate mixtures of pyroxene, amorphous carbon, and serpentine. As before, the low albedo is achieved by the use of the carbon, while in these models the pyroxene imparts the red color. In the models in the bottom panel of Fig. 4, graphite is included; this component has a slightly blue color at the long wavelengths.

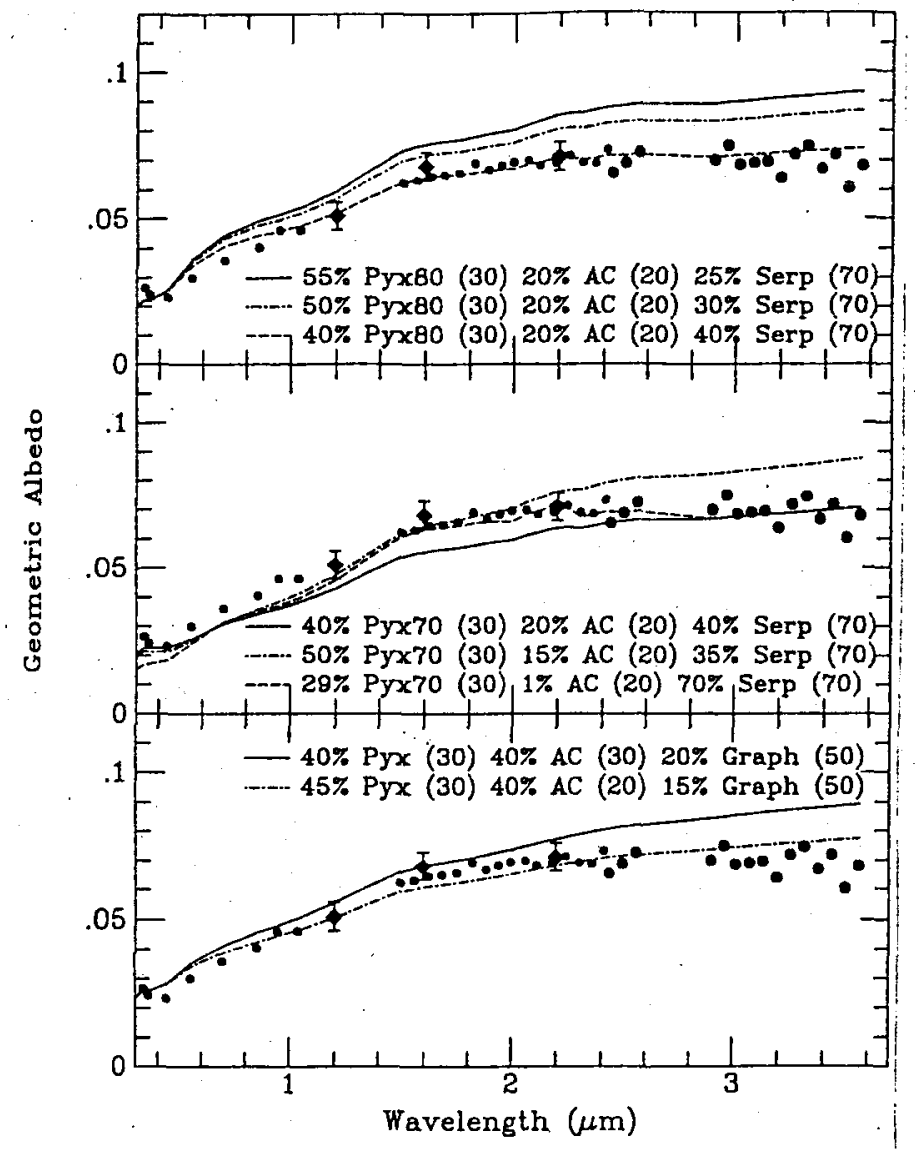

Figure 5. Models of D-type asteroid 624 Hektor without organics. Pyx $80=$ pyroxene with $0.8 \mathrm{Mg}$, Pyx70 = pyroxene with $0.7 \mathrm{Mg}, \mathrm{AC}=$ amorphous carbon, Serp $=$ serpentine, Graph = graphite. Grain sizes are given in parentheses. These are all intimate mixtures, with the proportions shown as percentages. Adapted from Cruikshank et al. (2001) 


\section{CONCLUSIONS}

We find that we can match the geometric albedos and colors of the reddest color groupings of outer Solar System bodies with models computed with scattering theory and the use of optical properties of synthetic organic solids (tholins), particularly Ice Tholin II and Titan tholin. In our models, elemental amorphous carbon must be added to the organic solids to achieve the low geometric albedos observed or assumed for these objects. The presence of elemental carbon can occur in nature by the dehydrogenation of tholins. In the use of tholins to achieve the observed red colors, our results are consistent with those of several other investigators, including the recent work of Dotto et al. (2003) and Doressoundiram et al. (2003). The least red objects in the outer Solar System can be modeled with mafic minerals, and do not require the presence of organic solids (Cruikshank et al. 2001).

We have incorporated into our models the space-weathered minerals found on the lunar surface. The Moon, Mercury, and many asteroids are reddened by sputtering of iron from iron-bearing minerals, and the deposition of neutral $\mathrm{Fe}$ on grains in the uppermost regolith. Our models of the reddest bodies in the outer Solar System do not require this mechanism to achieve the observed albedos and colors, although such space-weathered minerals may occur in modest quantities in the optically accessible surfaces.

\section{Acknowledgements}

The authors were supported in part by a NASA Planetary Astronomy grant (RTOP 344-32-20-01) We thank Dr. Ted Roush for making the Hapke code available, Dr. F. Poulet for making the Shkuratov modeling code available, and Dr. Yu. Shkuratov for providing the optical constants for lunar soil.

\section{Notes}

1 The number of significant figures in some of the modeling parameters suggests a degree of sensitivity of the fits to the data that is perhaps misleading. While the quality of fit of the preferred models is in fact sensitive to these parameters at a high level of accuracy, the models themselves have many more physical parameters that are not well known for most bodies in the Solar System. In addition, the complex refractive indices of the materials we use in the models are in some cases uncertain by $30 \%$ or more. Once a set of refractive indices is selected and other parameters of particle scattering are chosen, the calculated models are quite sensitive to the abundances of the components, their grain sizes, and their mixing geometry. 


\section{References}

Andronico, G., Baratta, G. A., Spinella, F., and Strazzulla, G. Optical evolution of laboratory-produced organics: Applications to Phoebe, Iapetus, outer belt asteroids, and cometary nuclei. Astron. Astrophys. 184, 333-336, 1987.

Barucci, M. A., Fulchignoni, M., Birlan, M., Doressoundiram, A., Romon, J., and Boehnhardt, H. Analysis of trans-Neptunian and Centaur colours: Continuous trend or grouping? Astron. Astrophys. 371, 1150-1154, 2001.

Binzel, R. The optical spectrum of 5145 Pholus. Icarus 99, 238-240, 1992.

Bradley, J. P., Keller, L. P., Brownlee, D. E., and Thomas, K. L. Reflectance spectroscopy of interplanetary dust particles. Met. Planet. Sci. 31, 394-402, 1996.

Brown, R. H., Cruikshank, D. P., Pendleton, Y. J., and Veeder G. J. Identification of water ice on the Centaur 1997 CU26. Science 280, 1430-1432, 1998.

Brown, R. H., Cruikshank, D. P., and Pendleton, Y. J. Water ice on Kuiper Belt object 1996 TO66. Astrophys. J. Lett. 519 L101-L104, 1999.

Brucato, J., Strazzulla, G., Baratta, G., Minella, V., and Colangeli, L. Laboratory studies on silicates relevant for the physics of TNOs. This volume, 2003.

Cooper, J. F., Christian, E.R., and Johnson, R. E. This volume, 2003.

Cronin, J. R., and Chang, S. Organic matter in meteorites: Molecular and isotopic analyses of the Murchison meteorite. In Greenberg, J. M., Mendoza-Gomez, C. X., and Pirronello (eds.) The Chemistry of Life's Origins, Kluwer, 209-258, 1993

Cronin, J. R., Pizzarello, S., and Cruikshank, D. P. Organic matter in carbonaceous chondrites, planetary satellites, asteroids and comets. In Meteorites and the Early Solar System (J. F. Kerridge and M. S. Matthews, Eds.), pp. 819-857. Univ. of Arizona Press, Tucson, 1988

Cruikshank, D. P., Roush, T. L., Bartholomew, M. J., Geballe, T. R., Pendleton, Y. J., White, S. M., Bell, J. F. III, Davies, J. K., Owen, T. C., de Bergh, C., Tholen, D. J., Bernstein, M. P., Brown, R. H., Tryka, K. A., and Dalle Ore, C. M. The composition of centaur 5145 Pholus. Icarus 135, 389-407, 1998.

Cruikshank, D. P. and Khare, B. N. Planetary Surfaces of Low Albedo: Organic Material Throughout the Solar System. In Bioastronomy '99-A New Era in Bioastronomy (G. A. Lemarchand and K. J. Meech, Eds.), pp. 253-261. Astron. Soc. Pacific Conf. Series, 2000.

Cruikshank, D. P., Dalle Ore, C. M., Roush, T. L., Geballe, T. R., Owen, T. C., de Bergh, C., Cash, M. D., and Hartmann, W. K. Constraints on the composition of Trojan asteroid 624 Hektor. Icarus 153, 348-360, 2001.

Davies, J. K., Sykes, M. V., and Cruikshank, D. P. Near-infrared photometry and spectroscopy of the unusual minor planet 5145 Pholus (1992AD). Icarus 102, 166-169, 1993.

Davies, J. K., Roush, T. L., Cruikshank, D. P., Bartholomew, M. J., Geballe, T. R., Owen, T., and de Bergh, C. The detection of water ice in comet Hale-Bopp. Icarus 127, 238-245, 1997.

Doressoundiram, A., Tozzi, G. P., Barucci, M. A., Boehnhardt, H., Fornasier, S., and Romon, J. ESO large programme on trans-Neptunian objects and Centaurs: Spectroscopic investigation of Centaur 2001 BL41 and TNOs (26181) 1996 GQ21 and (26375) 1999 DE9. Astron. J. 125, 2721-2727, 2003.

Dotto, E., Barucci, M. A., Boehnhardt, H., Romon, J., Doressoundiram, A., Peixinho, N., de Bergh, C., and Lazzarin, M. Searching for water ice on 471711999 
TC36, 1998 SG35, and 2000 QC243: ESO large program on TNOs and Centaurs. Icarus 162, 408-414, 2003.

Dotto, E., Barucci, M. A., and de Bergh, C. Colours and composition of the Centaurs. This volume, 2003.

Fink, U., M. Hoffmann, W. Grundy, M. Hicks, and W. Sears. The steep red spectrum of $1992 \mathrm{AD}$ : An asteroid covered with organic material? Icarus 97, 145-149, 1992.

Grundy, W. M., and Schmitt, B. The temperature-dependent near-infrared absorption spectrum of hexagonal $\mathrm{H}_{2} \mathrm{O}$ ice. J. Geophys. Res. 103, 25,809-25,822, 1998.

Hainaut, O. R., and Desanti, A. C. Colors of minor bodies in the outer Solar System. A statistical analysis. Astron. Astrophys 389, 641-664, 2002.

Hapke, B. Bidirectional reflectance spectroscopy, 1. Theory. J. Geophys. Res. 86, 3039-3054, 1981.

Hapke, B. Theory of Reflectance and Emittance Spectroscopy. 455 pp. Cambridge Univ. Press, New York, 1993.

Hapke, B. Space weathering from Mercury to the asteroid belt. J. Geophys. Res. 106, 10039-10074, 2001.

Imanaka, H., Khare, B. N., Elsila, J. E., Bakes, E. L. O., McKay, C. P., Cruikshank, D. P., Sugita, S., Matsui, and Zare, R. N. Chemical properties of Titan haze at various altitudes. (in preparation), 2003.

Jewett, D. C. From Kuiper Belt object to cometary nucleus: The missing ultrared matter. Astron. J. 123, 1039-1049, 2002.

Johnson, R. L. Effect of irradiation on the surface of Pluto. Geophys. Res. Lett. 16, 1233-1236, 1989.

Khare, B. N., Sagan, C., Arakawa, E. T., Suits, R., Callcot, T. A., and Williams, M. W. Optical constants of organic tholins produced in a simulated Titanian atmosphere: From soft $\mathrm{x}$-ray to microwave frequencies. Icarus 60, 127-137, 1984.

Khare, B. N., Thompson, W. R., Cheng, L., Chyba, C., Sagan, C., Arakawa, E. T., Meisse, C., and Tuminello, P. Production and optical constants of ice tholin from charged particle irradiation of $(1: 6) \mathrm{C}_{2} \mathrm{H}_{6} / \mathrm{H}_{2} \mathrm{O}$ at $77 \mathrm{~K}$. Icarus $103,290-300$, 1993.

Maurette, M., Engrand, C., and Kurat, G. Collection and microanalysis of Antarctic micrometeorites. In Physics, Chemistry, and Dynamics of Interplanetary Dust (B. A. A. Gustafson and M. S. Hanner, Eds.), pp 265-273, 1996. ASP Conf. Ser. 104.

McBride, N., Green, S. F., Davies, J. K., Tholen, D. J., Sheppard, S. S., Whiteley, R. J., and Hillier, J. K. Visible and infrared photometry of Kuiper Belt objects: Searching for evidence of trends. Icarus 161, 501-510, 2003.

McDonald, G. D., Thompson, W. R., Heinrich, M., Khare, B. N., and Sagan, C. Chemical investigation of Titan and Triton tholins. Icarus 108, 137-145, 1994.

McDonald, G. D., Whited, L. J., DeRuiter, C., Khare, B. N., Patniak, A., and Sagan, C. Production and chemical analysis of cometary ice tholins. Icarus 122, 107-117, 1996.

Mueller, B. E. A., Tholen, D.'J., Hartmann, W. K., and Cruikshank, D. P. Extraordinary colors of asteroidal object (5145) 1992 AD. Icarus 97, 150-154, 1992.

Noll, K., Stephens, D., Grundy, W., Millis, R., Buie, M., Spencer, J., Tegler, S., Romanishin, W., and Cruikshank, D. Discovery of trans-Neptunian binaries with the Hubble Space Telescope. Bull. A.A.S. 34, 849 (abstract), 2002. 
Owen, T. C., Cruikshank, D. P., Dalle Ore, C. M., Geballe, T. R., Roush, T. L., and de Bergh, C. Detection of water ice on Saturn's satellite Phoebe. Icarus 139, 379-382, 1999.

Pieters, C. M., L. A. Taylor, S. K. Noble, L. P. Keller, B. Hapke, R. V. Morris, C. C. Allen, D. S. McKay, and S. Wentworth. Space weathering on airless bodies: Resolving a mystery with lunar samples. Meteorit. Planet. Sci. 35, 1101-1107, 2000.

Poulet, F., Cuzzi, J.N., Cruikshank, D. P., Roush, T., and Dalle Ore, C. M. Comparison between the Shkuratov and Hapke scattering theories for solid planetary surfaces. Application to the surface composition of two centaurs. Icarus 160 , $313-324,2002$.

Ramirez, S. I.; Coll, P.; da Silva, A.; Navarro-Gonzlez, R.; Lafait, J.; Raulin, F. Complex refractive index of Titan's aerosol analogues in the 200-900 $\mathrm{nm}$ domain. Icarus 156, 515-529, 2002.

Shkuratov, Y., Starukhina, L., Hoffmann, H., and Arnold, G. A model of spectral albedo of particulate surfaces: Implications for optical properties of the Moon. Icarus 137, 235-246, 1999.

Thompson, W. R., Murray, B. G. J. P. T., Khare, B. N., and Sagan, C. Coloration and darkening of methane clathrate and other ices by charged particle irradiation: Applications to the outer Solar System. J. Geophys. Res., 92, 14,933-14,947, 1987.

Tokunaga, A. T. Infrared astronomy. In Cox, A. N. (Ed.), Allen's Astrophysical Quantities, Springer-Verlag, New York, pp. 143-167, 2000.

Tran, B. N., Ferris, J. P., and Chera, J. J. The photochemical formation of a Titan haze analog. Structural analysis by $X$-ray photoelectron and infrared spectroscopy. Icarus 162, 114-124, 2003.

Wooden, D. H., Butner, H. M., Harker, D. E., and Woodward, C. E. Mg-rich silicate crystals in comet Hale-Bopp: ISM relics or solar nebula condensates? Icarus, 143, 126-137, 2000. 